\title{
Pediatric Drug-Induced Sleep Endoscopy: Technique and Scoring System
}

\author{
Adrian Williamson $\mathrm{IV}^{1}$, Samira R. Ibrahim ${ }^{2}$, Steven W. Coutras ${ }^{1}$, Michele M. Carr ${ }^{3}$ \\ 1. Department of Otolaryngology, Head and Neck Surgery, West Virginia University, Morgantown, USA 2. Department \\ of Otolaryngology, Head and Neck Surgery, West Virginia School of Osteopathic Medicine, Lewisburg, USA 3. \\ Department of Otolaryngology, Head and Neck Surgery, University at Buffalo, Buffalo, USA
}

Corresponding author: Michele M. Carr, mmcarr2001@gmail.com

\begin{abstract}
Drug-induced sleep endoscopy (DISE) is an invaluable tool for identifying sites of obstruction for patients with obstructive sleep apnea (OSA). During DISE, the patient is in a state of drug-induced sleep, and a flexible laryngoscope is passed through the nose into the upper airway. Sites of obstruction are visualized and scored to guide surgical management. Currently, there is no universally accepted method of DISE analysis and scoring. This limitation in comparability impedes large-scale analysis between clinicians, institutions, and studies. In this report, we propose a standardized method of scoring and performing DISE in children with OSA. Our DISE scoring system is internally developed, consistent through the study, and addresses all levels of potential upper airway obstruction.
\end{abstract}

Categories: Otolaryngology, Pediatrics

Keywords: drug induced sleep endoscopy, pediatric obstructive sleep apnea, sleep apnea surgery, dise, osa, tonsillectomy, adenoidectomy

\section{Introduction}

Obstructive sleep apnea (OSA) is a common childhood syndrome that can be associated with significant morbidity and is known to be detrimental to neurobehavioral, cardiovascular, endocrine, and metabolic health and development in children [1]. The prevalence of OSA is between $1 \%$ and $4 \%$, however, it is likely under-diagnosed and under-treated [2,3]. The diagnostic gold standard for OSA is overnight polysomnography (PSG); however, this does not provide anatomic information related to the cause of airway obstruction.

Drug-induced sleep endoscopy (DISE) in children is a useful tool for identifying sources of obstruction and planning future surgical intervention. During this procedure, the patient is in a state of drug-induced sleep, and a flexible laryngoscope is passed through the nose into the upper airway. Sites of obstruction are visualized and documented, and this information is used to guide surgical management. In general, DISE demonstrates statistical promise in terms of safety, validity, test-retest reliability, and inter/intra-rater reliability [4-7]. The purposed indications for DISE in children include documented persistent OSA after tonsillectomy and adenoidectomy, high risk for persistent OSA after tonsillectomy and adenoidectomy, significant sleep-disordered breathing (SDB) or OSA in patients with small tonsils and adenoids, concern for occult or sleep-state dependent laryngomalacia, and to confirm the level of obstruction prior to placement of hypoglossal nerve stimulator [5].

Williamson et al. This is an open access article distributed under the terms of the Creative Commons Attribution License CC-BY 4.0., which permits unrestricted use, distribution, and reproduction in any medium, provided the original author and source are credited.

There are several published scoring systems, however, none are used universally. The lack of consensus prevents objective outcome analysis between clinicians, institutions, and studies [8]. This limitation in comparability is important to consider as it may impede large-scale analysis such as systematic reviews and meta-analyses of refractory OSA patients. Commonly used DISE scoring systems differ in how many sites of obstruction are evaluated, description of airway narrowing, and obstruction configurations [5,9]. In this report, we propose a standardized method of scoring and performing DISE in children with refractory OSA. This comprehensive and easy to follow method takes into account all potential sites of obstruction, degree of narrowing, and obstruction configuration.

\section{Technical Report}

DISE is performed in the operating room with a pediatric anesthesia team that provides the necessary sedation. Following standard protocol for pediatric patients, anesthesia is first induced using an inhaled anesthetic agent allowing for intravenous access to be obtained. The inhalation agent is then discontinued and a propofol infusion dosed appropriately for the child's age and weight is used for the remainder of the procedure. The patients are monitored closely while placed in supine position with the chin in neutral position.

DISE is initiated when audible or palpable snoring is noted. The flexible fiberoptic laryngoscope is passed 


\section{Cureus}

into the nose bilaterally. The flexible laryngoscope is advanced into the nasopharynx, followed by the oropharynx, and ends at the level of the supraglottic larynx. The airway patency is evaluated throughout the exam. The DISE procedures are recorded in video form and still images are taken during the procedure for later review and documentation (Figure 1). The DISE procedure is documented using a uniform template and scoring system (Figure 1). The scoring system records the operator's evaluation of the bilateral nasal airway, adenoid, retropalatal airway, oropharyngeal airway, retrolingual airway, and laryngeal airway separately. Obstruction at each site or by the specified structure is broadly categorized as non-obstructive, partially obstructive, or significantly obstructive (Figure 1). The completed template results in a narrative record of the DISE findings for that case.

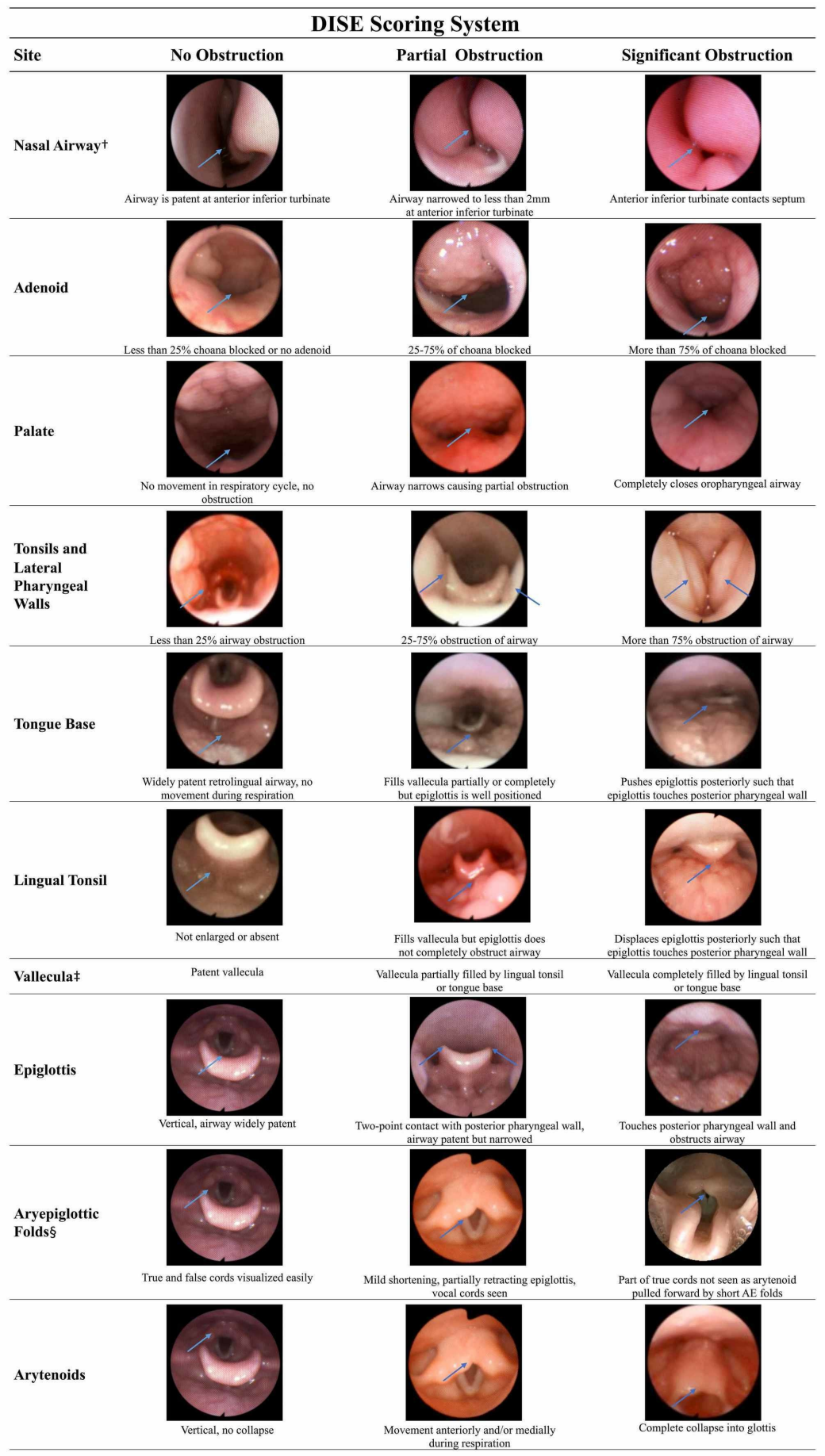

\section{FIGURE 1: Drug-induced sleep endoscopy (DISE) scoring system}

This figure depicts the scoring system used with overlying picture examples. †Only left turbinate example pictures are provided, however, in practice the right and left nasal airway documented separately; $\neq$ For vallecula example pictures, see tongue base and lingual tonsil rows. §Aryepiglottic folds were visualized with and without chin lift during DISE. 


\section{Cureus}

\section{Discussion}

The scope of DISE research is limited by lack of consistent quantitative data and range of methodology used among institutions and otolaryngologists. The adoption of a standard method and scoring system for pediatric DISE would help remedy this constraint on DISE research. The most obvious limitation is the lack of consistent scoring systems among studies and institutions. Six different scoring systems (Bachar, Boudewns, Chan, Fishman, Sleep Endoscopy Rating Scale (SERS), Velum, Oropharynx and lateral pharyngeal walls, Tongue bass and Epiglottis (VOTE)) have been used to report pediatric DISE findings (Table 1) [10-15]. The goal of all these scoring systems is to concisely and consistently communicate findings during DISE in a manner that can be quantified and studied. However, these systems are each unique with regard to the anatomic sites included in the system and in their manner of quantifying and characterizing airway

DISE scoring systems used in children.

\section{Scoring Sites}

System

1. Nasal airway

and

Nasopharynx

\section{Palate and}

Bachar et tonsils

al. 2012

[10]

3. Tongue

base

4.

Hypopharynx

5. Larynx

1. Adenoid

2. Palate

2. Tonsils and

Boudewyns oropharynx

et al. 2014

[11]

3. Tongue

base

4. Epiglottis

5. Larynx

1. Adenoid

2. Velum

3. Lateral pharyngeal

Chan et al. walls and

2014 [12] oropharynx
4. Tongue
base
5. Epiglottis
and

\section{Quantification of obstruction}

Additional comments

Score of 1 or 2 given for partial or complete obstruction respectively

Score of 0 for no obstruction, 1 for less than $50 \%$ obstruction, 2 for between $50-75 \%$ obstruction, and 3 for greater than $75 \%$ obstruction Score of 0 for no collapse, 1 for collapse present

Score of 0 for no obstruction, 1 for less than $50 \%$ obstruction, 2 for between $50-90 \%$ obstruction, and 3 for tonsils that touch at midline.

Score of 0 for no obstruction, 1 for partial obstruction, 2 for complete obstruction

Score of 0 for no collapse, 1 for collapse present

Laryngomalaica is noted to be absent (score of 0 ) or present (score of 1)

Score of 0 for no obstruction, 1 for less than $50 \%$ obstruction, 2 for between $50-99 \%$ obstruction, and 3 for complete obstruction
This grading system converts to a NTPHL staging system. Sites without obstruction are not included in the staging for that patient.
This system includes a general impression of hypotonia as present or absent. 


\section{Cureus}

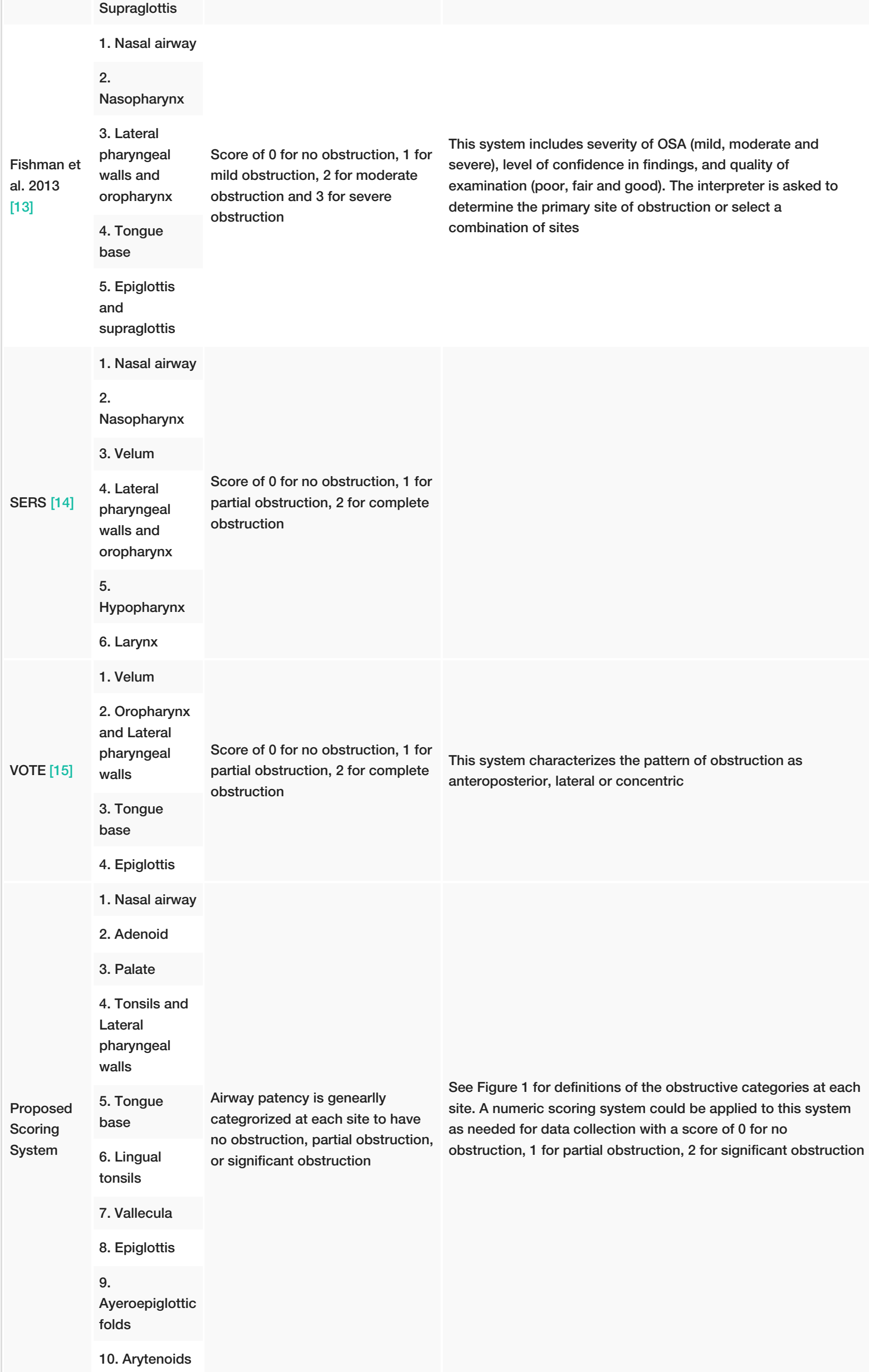

Score of 0 for no obstruction, 1 for partial obstruction, 2 for complete obstruction

This system includes severity of OSA (mild, moderate and severe), level of confidence in findings, and quality of examination (poor, fair and good). The interpreter is asked to determine the primary site of obstruction or select a

$\begin{array}{ll}\text { obstruction } & \text { determine the primary } \\ \text { combination of sites }\end{array}$

This system characterizes the pattern of obstruction as anteroposterior, lateral or concentric

See Figure 1 for definitions of the obstructive categories at each site. A numeric scoring system could be applied to this system as needed for data collection with a score of 0 for no obstruction, 1 for partial obstruction, 2 for significant obstruction
Airway patency is genearly categrorized at each site to have no obstruction, partial obstruction, or significant obstruction 
The VOTE system is the most studied scoring system and has been used in both children and adults [15]. The system is concise and easy to use. However, a major limitation of the VOTE scoring system for use in children is the omission of the nasopharyngeal and supraglottic sites. The Chan scoring system is similar to the VOTE system but includes the supraglottis and lingual tonsils specifically, which are important sources of obstruction in children $[12,15]$. The Chan system uses a scoring system which correlates to the percentage of obstruction at all sites except the lingual tonsil which is described only as present or absent [12]. The Bachar system and SERS are unique in that they are an overall score of upper airway obstruction $[10,14]$. The Boudewyns system is unique in that it includes a designation of generalized hypotonia as present or absent and characterizes whether the obstruction is fixed or dynamic [11]. The Fishman system evaluates the degree of obstruction at several upper airway subsites but it is unique in that it also notes the quality of the exam, confidence in the findings, and OSA severity [13]. While the validity and reliability of DISE is promising, the clinical significance of these scoring systems is not well established [5-7]. Our DISE scoring system attempts to evaluate all levels of potential airway obstruction while maintaining a simple and easy to follow guide. The ideal scoring system should be simple and practical with proven reliability and should completely characterize the nature of obstruction in order to guide management.

Another concern with DISE research is how accurately intravenous anesthesia simulates normal sleep for the patient. It is important to note that all anesthetics used for DISE have some documented effect on sleep architecture [5,16]. The anesthetic-specific effect on the degree or level of obstruction has not been well studied. A combination of dexmedetomidine and ketamine is preferred by some pediatric sleep surgeons due to the lower risk of respiratory depression and upper airway obstruction as seen with other agents $[5,17]$. In this protocol, propofol is used which has a more rapid onset and shorter duration as compared to dexmedetomidine. Propofol can result in deeper sedation and more pronounced airway collapse while dexmedetomidine provides longer lasting sedation with little effect on upper airway obstruction [16,18-20]. During DISE, it is critical that the depth of sedation is closely monitored in coordination with the anesthesiologist. There is an ongoing debate regarding the best anesthetic protocol for DISE and more research with direct comparison of these agents is needed.

\section{Conclusions}

The future clinical value of pediatric DISE depends on the continuation of ongoing research and development of future studies. At this time large-scale institutional studies, systematic reviews, and metaanalyses which could improve the power of DISE research are limited by the lack of a universally accepted technique and scoring system. Here, we have proposed a specific protocol for scoring of DISE in children. More research is needed to determine the reliability of scoring systems used for DISE and to determine the optimal anesthetic protocol used during DISE.

\section{Additional Information \\ Disclosures}

Human subjects: Consent was obtained by all participants in this study. West Virginia University Office of Research Integrity and Compliance issued approval 1706614679R001. Animal subjects: All authors have confirmed that this study did not involve animal subjects or tissue. Conflicts of interest: In compliance with the ICMJE uniform disclosure form, all authors declare the following: Payment/services info: All authors have declared that no financial support was received from any organization for the submitted work. Financial relationships: All authors have declared that they have no financial relationships at present or within the previous three years with any organizations that might have an interest in the submitted work. Other relationships: All authors have declared that there are no other relationships or activities that could appear to have influenced the submitted work.

\section{References}

1. Capdevila OS, Kheirandish-Gozal L, Dayyat E, Gozal D: Pediatric obstructive sleep apnea: complications, management, and long-term outcomes. Proc Am Thorac Soc. 2008, 5:274-82.

2. DelRosso LM: Epidemiology and diagnosis of pediatric obstructive sleep apnea. Curr Probl Pediatr Adolesc Health Care. 2016, 46:2-6. 10.1016/j.cppeds.2015.10.009

3. Chervin R, Archbold K, Panahi P, Pituch K: Sleep problems seldom addressed at two general pediatric clinics. Pediatrics. 2001, 107:1375-1380. 10.1542/peds.107.6.1375

4. Ravesloot MJ, de Vries N: One hundred consecutive patients undergoing drug-induced sleep endoscopy: results and evaluation. Laryngoscope. 2011, 121:2710-2716. 10.1002/lary.22369

5. Wilcox LJ, Bergeron M, Reghunathan S, Ishman SL: An updated review of pediatric drug-induced sleep 
endoscopy. Laryngoscope Investig Otolaryngol. 2017, 2:423-431. 10.1002/lio2.118

6. Rodriguez-Bruno K, Goldberg AN, McCulloch CE, Kezirian EJ: Test-retest reliability of drug-induced sleep endoscopy. Otolaryngol Head Neck Surg. 2009, 140:646-651. 10.1016/j.otohns.2009.01.012

7. Kezirian EJ, White DP, Malhotra A, Ma W, McCulloch CE, Goldberg AN: Interrater reliability of drug-induced sleep endoscopy. Arch Otolaryngol Head Neck Surg. 2010, 136:393-397. 10.1001/archoto.2010.26

8. Nzekwu C, Dijemeni E: Drug-induced sleep endoscopy (DISE) scoring systems: ideal DISE scoring system and comparability properties. Otolaryngol Head Neck Surg. 2018, 158:777. 10.1177/0194599817753603

9. Amos JM, Durr ML, Nardone HC, Baldassari CM, Duggins A, Ishman SL: Systematic review of drug-induced sleep endoscopy scoring systems. Otolaryngol Head Neck Surg. 2018, 158:240-248. 10.1177/0194599817737966

10. Bachar G, Nageris B, Feinmesser R, et al.: Novel grading system for quantifying upper-airway obstruction on sleep endoscopy. Lung. 2012, 190:313-318. 10.1007/s00408-011-9367-3

11. Boudewyns A, Verhulst S, Maris M, Saldien V, Van de Heyning P: Drug-induced sedation endoscopy in pediatric obstructive sleep apnea syndrome. Sleep Med. 2014, 15:1526-1531. 10.1016/j.sleep.2014.06.016

12. Chan DK, Liming BJ, Horn DL, Parikh SR: A new scoring system for upper airway pediatric sleep endoscopy . JAMA Otolaryngol Head Neck Surg. 2014, 140:595-602. 10.1001/jamaoto.2014.612

13. Fishman G, Zemel M, DeRowe A, Sadot E, Sivan Y, Koltai PJ: Fiber-optic sleep endoscopy in children with persistent obstructive sleep apnea: inter-observer correlation and comparison with awake endoscopy. J Pediatr Otorhinolaryngol. 2013, 77:752-755. 10.1016/j.ijporl.2013.02.002

14. Lam DJ, Weaver EM, Macarthur CJ, et al.: Assessment of pediatric obstructive sleep apnea using a druginduced sleep endoscopy rating scale. Laryngoscope. 2016, 126:1492-1498. 10.1002/lary.25842

15. Kezirian EJ, Hohenhorst W, de Vries N: Drug-induced sleep endoscopy: the VOTE classification. Eur Arch Otorhinolaryngol. 2011, 268:1233-1236. 10.1007/s00405-011-1633-8

16. Ehsan Z, Mahmoud M, Shott SR, Amin RS, Ishman SL: The effects of anesthesia and opioids on the upper airway: a systematic review. Laryngoscope. 2016, 126:270-84. 10.1002/lary.25399

17. Shteamer JW, Dedhia RC: Sedative choice in drug-induced sleep endoscopy: a neuropharmacology-based review. Laryngoscope. 2017, 127:273-279. 10.1002/lary.26132

18. Crawford MW, Rohan D, Macgowan CK, Yoo SJ, Macpherson BA: Effect of propofol anesthesia and continuous positive airway pressure on upper airway size and configuration in infants. Anesthesiology. 2006, 105:45-50. 10.1097/00000542-200607000-00011

19. Mahmoud M, Gunter J, Donnelly LF, Wang Y, Nick TG, Sadhasivam S: A comparison of dexmedetomidine with propofol for magnetic resonance imaging sleep studies in children. Anesth Analg. 2009, 109:745-53. 10.1213/ane.0b013e3181adc506

20. Mahmoud M, Radhakrishman R, Gunter J, et al.: Effect of increasing depth of dexmedetomidine anesthesia on upper airway morphology in children. Paediatr Anaesth. 2010, 20:506-15. 10.1111/j.14609592.2010.03311.x 